\title{
Aplicação de sondagens elétricas verticais na estimativa de contatos entre unidades geológicas nos municípios de São João de Pirabas e Salinópolis, região costeira do nordeste do Pará. Monteiro*, D. P., UFPA, Silva, D., UFPA, Aguirre, H. R. B., UFPA, Soares, J. L., UFPA
}

Copyright 2018, SBGf - Sociedade Brasileira de Geofísica

Este texto foi preparado para a apresentação no VIII Simpósio Brasileiro de Geofísica, Salinópolis, 18 a 20 de setembro de 2018. Seu conteúdo foi revisado pelo Comitê Técnico do VIII SimBGt, mas não necessariamente representa a opinião da SBGf ou Técnico do VIII SimBGt, mas não necessariamente representa a opinião da SBGf ou
de seus associados. É proibida a reprodução total ou parcial deste material para propósitos comerciais sem prévia autorização da SBGf.

\begin{abstract}
In the northeastern costal region of the state of Pará, there are Miocene deposits, designated Pirabas and Barreiras Formations, the term Pirabas Formation being used for carbonate sedimentation and Barreiras Formation is used for siliciclastic deposits the superimposed. The apparent resistivity data was processed using the IPI2win program (Geoscan-M Ltda) designed to perform automatic 1D inversion, correlating them with lithological profiles of wells, available in the online platform of the Groundwater Information System (SIAGAS). The results obtained showed satisfactory, to identify the contact between the Formations, the top of the crystalline basement and the identification of subterranean aquifers.
\end{abstract}

Keywords: Electrical resistivity, Vertical electrical soundings (VES), Miocene deposits.

\section{Introdução}

Na região nordeste do Pará ocorre unidades carbonáticas e siliciclásticas neógenas representativas das Formações Pirabas (Mioceno inferior), Barreiras (Mioceno médio a Plioceno) e sedimentos quaternários da unidade PósBarreiras que compõem a Plataforma Bragantina (Rossetti et al., 2001). A deposição destas unidades foi particularmente controlada pela formação e reativação de falhas normais e transcorrentes (Costa, 1996), como também por mudanças eustáticas e climáticas que geraram flutuações do nível relativo do mar (Rossetti, 2001).

A Formação Pirabas é caracterizada por sedimentação carbonática contendo margas, biocalcarenito estratificados ou não estratificados, biocalcirudito, biocaciluditos, folhelhos negros e cinza esverdeada, além de abundante conteúdo fossilífero como conchas, foraminíferos e restos de vegetais com concreções de pirita (Petri, 1954, 1957; Ferreira, 1966; Góes et al., 1990; Rossetti; Góes, 2004) e a Formação Barreiras é formada unicamente de sedimento siliciclásticos, constituídos por uma variedades de rochas onde se destacam arenitos maciços, pelitos e conglomerados (Rossetti et al., 1989), com pouco conteúdo fossilífero.
Os principais estudos nestas unidades restringem-se ao conteúdo paleontológico e análise faciológica (Rossetti, 2006; Góes et al., 1990), além do grande número de poços tubulares existentes na região, que disponibilizam informações litológicas pontuais. Porém, estes estudos têm-se mostrado insuficientes para o conhecimento da disposição em subsuperfície dos contatos entre as Formações Barreiras e Pirabas e do embasamento cristalino.

Um método economicamente viável e eficaz para estimar tanto as disposições dos contatos entre as Formações como o topo do embasamento cristalino é o método da eletrorresistividade, que se baseia no estudo da diferença de potencial criado pela injeção de corrente elétrica no terreno. Conhecendo-se a corrente injetada e a diferença de potencial pode-se calcular a resistividade aparente, que está relacionada à geologia do ambiente investigado.

Com o método foram coletadas medidas de corrente e potencial, utilizando sondagens elétricas verticais (SEVs) em perfis de $630 \mathrm{~m}$ em São João de Pirabas, na Vila de Santa Luzia e $540 \mathrm{~m}$ em Salinópolis, próximo à praia do Atalaia, com os eletrodos dispostos de acordo com o arranjo Wenner, o qual apresenta relativa sensibilidade à variações verticais de resistividade, ou seja estruturas horizontais, segundo Loke (1999).

Neste artigo apresentamos a eficiência do uso do método da eletrorresistividade na identificação dos contatos entre as Formações Barreiras e Pirabas e o topo do embasamento cristalino, nos municípios de São João de Pirabas e Salinópolis, utilizando informações geológicas a priori de perfis de poços disponíveis na plataforma online de Sistema de Informação de Águas Subterrâneas (SIAGAS).

\section{Caracterização da área}

As áreas de estudo localizam-se geograficamente na região costeira do nordeste do Pará, aos arredores da PA-124, nos municípios de São João de Pirabas e Salinópolis (Figura 1), onde ocorre um extenso registro Miocênico favorecido excepcionalmente pela preservação de abundantes estruturas sedimentares, representadas pelas Formações litoestratigráficas Pirabas e Barreiras (Rossetti, 2000, 2001, 2006; Rossetti; Santos Jr., 2004). Os municípios de São João de Pirabas e Salinópolis estão situados as latitude $00^{\circ} 46^{\prime} 47^{\prime \prime}$ Sul e longitude $47^{\circ} 10^{\prime} 52^{\prime \prime}$ Oeste e $00^{\circ} 36^{\prime} 49^{\prime \prime}$ Sul e longitude $47^{\circ} 21^{\prime} 22$ " Oeste, respectivamente. 


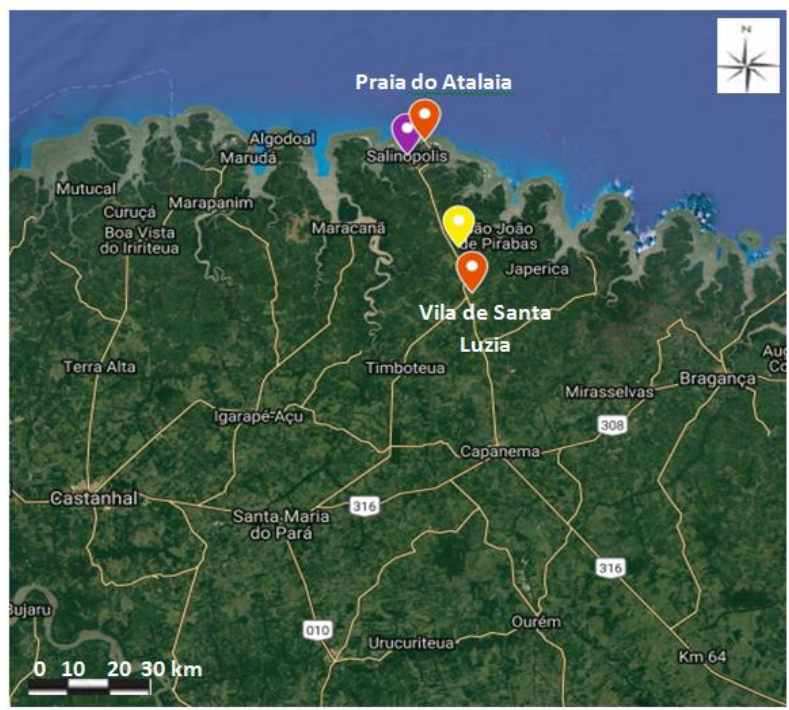

Figura 1- Região nordeste do Pará nos municípios de São João de Pirabas e Salinópolis.

\section{Método da eletrorresistividade}

Pertencente ao grupo dos métodos geoelétricos, a eletrorresistividade, é um método geofísico cujo princípio está baseado na determinação da resistividade elétrica aparente dos materiais existentes no ambiente geológico. O método consiste na fixação de quatro eletrodos no solo, dois de corrente $(A$ e $B)$ e dois de potencial $(M$ e $M)$, onde se injeta corrente pelos eletrodos $A$ e $B$, e então, realizam-se as medidas da diferença de potencial entre os eletrodos $M$ e $N$. Com a diferença de potencial é possível calcular a resistividade aparente do meio geológico investigado, que não é propriamente um parâmetro físico do meio e sim um valor integrado do semi-espaço sobre o qual a medida é efetuada, definida como

$$
\rho_{a}=K \frac{\Delta V}{l},
$$

onde $\rho_{a}$ é a resistividade aparente $[\Omega . \mathrm{m}], \Delta V$ é a diferença de potencial medida entre os eletrodos $M$ e $N$ [Volt], $I$ é a corrente aplicada entre os eletrodos $A$ e $B$ [Amper] e $K$ é o fator geométrico para o arranjo dos eletrodos AMNB, definido por

$$
K=2 \pi\left(\frac{1}{\overline{A M}}-\frac{1}{\overline{B M}}-\frac{1}{\overline{A N}}+\frac{1}{\overline{B N}}\right)^{-1},
$$

onde $\overline{A M}, \overline{A N}, \overline{B M}$ e $\overline{B N}$ são as distâncias entre os eletrodos.

No método da eletrorresistividade utilizam-se duas principais técnicas de investigação, o caminhamento elétrico, em que as medidas de resistividade aparente são realizadas ao longo de perfis, deslocando-se lateralmente os quatro eletrodos sem alterar seus espaçamentos e a sondagem elétrica vertical (SEV) que investiga a variação vertical da resistividade em profundidade, conseguida através do aumento da separação dos eletrodos de corrente. Nesta técnica, os eletrodos de corrente são normalmente deslocados simetricamente em relação a um ponto central, abaixo do qual se considera a amostragem de resistividade aparente.

Para estimar os contatos entre as Formações e topo do embasamento, utilizamos a técnica de sondagem elétrica vertical, com os eletrodos dispostos em arranjo Wenner (Figura 2), em que os quatro eletrodos são dispostos em linha e igualmente espaçados, sendo denominado o espaçamento de $a$, que é gradualmente aumentado ao redor de um ponto fixo central, aumentando-se a profundidade de investigação. Este arranjo foi utilizado devido às vantagens de possuir elevada razão sinal/ruído e relativa sensibilidade a estruturas horizontais, por exemplo, contatos geológicos. A resistividade aparente para este arranjo é definida como:

$$
\rho_{a}=2 \pi a \frac{\Delta V}{l}
$$

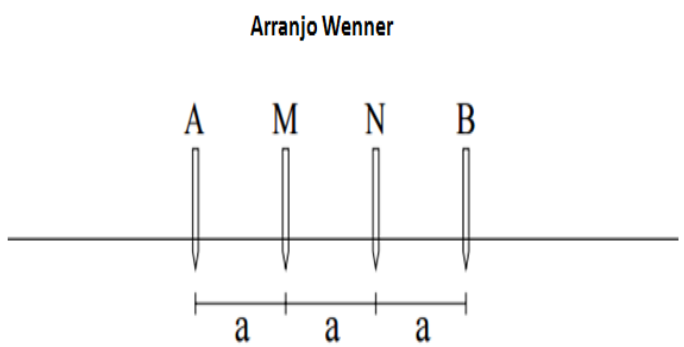

Figura 2 - Configuração do arranjo Wenner. $A$ e $B$ eletrodos de corrente e $\mathrm{M}$ e $\mathrm{N}$ eletrodos de potencial.

O equipamento utilizado para a aquisição dos dados foi o Resistivímetro Geotest Rd-1000, pertencente ao departamento de geofísica da Universidade Federal do Pará (Figura 3), composto por uma unidade transmissora $(T X)$ alimentada por duas baterias de 12 volts em série, que permite o ajuste da voltagem de saída entre 5 a 800 volts e outra receptora $(R X)$ alimentada por oito pilhas alcalinas de 1,5 volts.

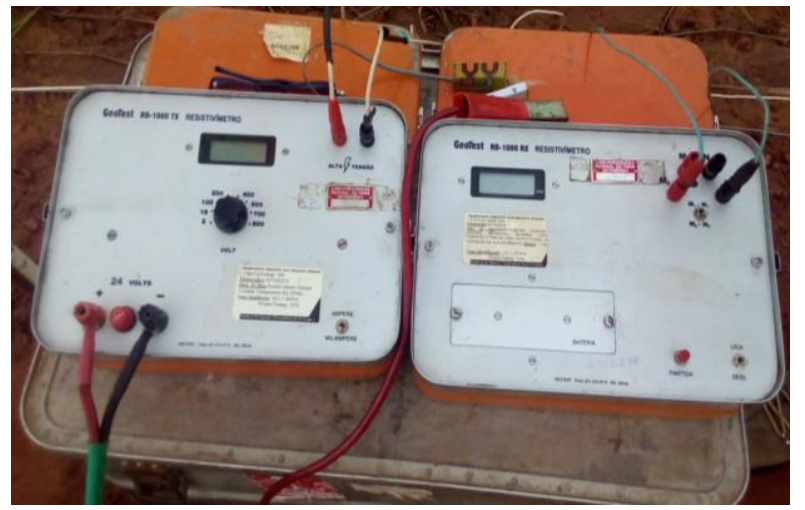

Figura 3 - Equipamento Resistivímetro Geotest RD-1000. 


\section{Resultados e interpretações}

Os dados das SEVs foram invertidos através do programa IPI2win (Geoscan-M Ltda) projetado para realizar inversões 1D automáticas de dados de eletrorresistividade, fornecendo informações importantes sobre a geologia local, como: resistividade, profundidade, espessura e altimetria, correspondente a um modelo provável de distribuição de resistividade em subsuperfície.

Para a escolha do modelo geoelétrico satisfatório utilizouse informações geológicas a priori de perfis de poços próximos às áreas de aquisição que auxiliaram na escolha do número de camadas do modelo, colaborando para um ajuste entre curvas satisfatório, em uma faixa de erro médio quadrático (root mean square - RMS) aceitável, que representa o erro de ajuste entre a curva de resistividade aparente de aquisição e a da resposta do modelo geoelétrico.

O resultado obtido na Vila de Santa Luzia representa um modelo geoelétrico (linha azul) de $70 \mathrm{~m}$ de profundidade, composto de sete camadas inseridas manualmente e um substrato. O ajuste possui um erro de $6,11 \%$ (Figura 4) e na tabela 1 mostra os parâmetros do modelo geoelétrico: resistividade $(\rho)$, espessura da camada $(h)$, profundidade (d) e altimetria $(A / t)$, que é a diferença de nível do modelo geoelétrico.

\section{SEV da Vila de Santa Luzia}

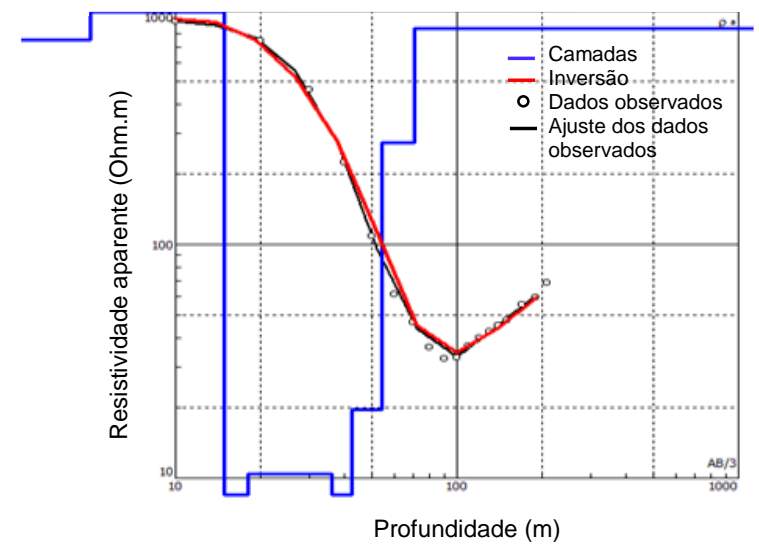

Figura 4-Curva de resistividade da Vila de Santa Luzia.

Tabela 1 - Resultado do modelo geoelétrico. $N$ : número de camadas, $\rho$ : resistividade, $h$ : espessura da camada, $d$ : profundidade e Alt: altimetria.

\begin{tabular}{|c|c|c|c|c|}
\hline \multirow{2}{*}{$\begin{array}{l}7 \\
\mathbf{N}\end{array}$} & \multicolumn{2}{|c|}{$\mathrm{RMS}=6.11 \%$} & \multirow[b]{2}{*}{ d } & \multirow[b]{2}{*}{ Alt } \\
\hline & $p$ & h & & \\
\hline 1 & 754 & 5 & 5 & -5 \\
\hline 2 & 1553 & 9.91 & 14.9 & -14.91 \\
\hline 3 & 3.24 & 3.2 & 18.1 & -18.1 \\
\hline 4 & 10.4 & 17.8 & 35.9 & -35.94 \\
\hline 5 & 7.66 & 6.39 & 42.3 & -42.33 \\
\hline 6 & 19.6 & 11.8 & 54.1 & -54.12 \\
\hline 7 & 273 & 16.5 & 70.6 & -70.61 \\
\hline 8 & 840 & & & \\
\hline
\end{tabular}

Com os resultados obtidos do modelo geoelétrico estabeleceu-se a correlação entre o perfil construtivo do poço da Vila de Santa Luzia e o modelo geoelétrico (Figura 5), obtendo as seguintes interpretações:

A primeira camada superficial de $5 \mathrm{~m}$ de espessura e alta resistividade igual a $754 \mathrm{Ohm} . \mathrm{m}$, corresponde ao solo rico em matéria orgânica e cascalho, com ausência de fluidos nos poros.

A segunda camada de 9,91 $\mathrm{m}$ de espessura e resistividade de $1553 \mathrm{Ohm} . \mathrm{m}$, refere-se a areia argilosa com intercalações de lateritas, correspondente à Formação Barreiras.

A partir da terceira camada apresenta-se a Formação Pirabas, caracterizada pela presença de sucessões carbonáticas, onde identificamos a localização do aquífero de 15 a $60 \mathrm{~m}$ de profundidade, evidenciado devido aos baixos valores de resistividade elétrica. As camadas são constituídas por: argila calcífera $(3,24$ Ohm.m), calcário fraturado (10,4 Ohm.m), argila verde $(7,66 \mathrm{Ohm} . \mathrm{m})$, areia grossa (19,6 Ohm.m) e folhelho (273 Ohm.m), respectivamente.

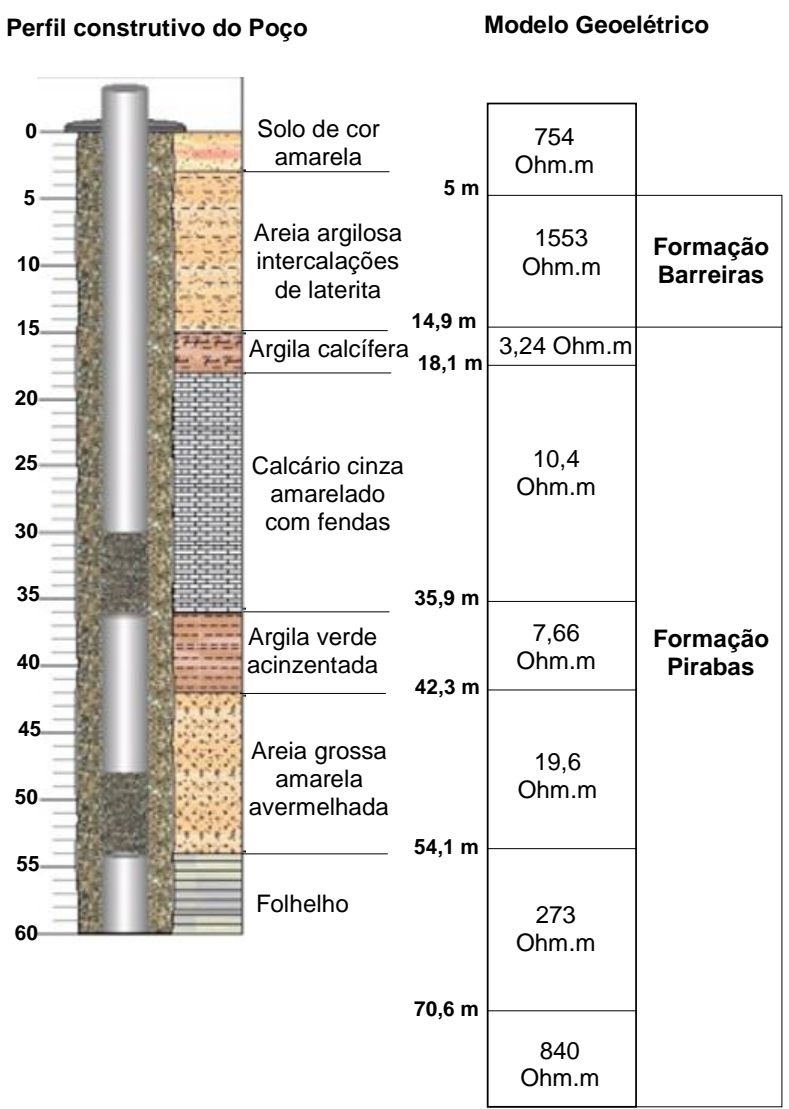

Figura 5 - Correlação entre o perfil construtivo do poço da Vila de Santa Luzia e o modelo geoelétrico.

A camada de folhelho e o substrato (840 Ohm.m) apresentam alta resistividade, possivelmente por não conterem fluidos intercalados nos poros constituintes das camadas. 
O resultado obtido próximo à praia do Atalaia representa um modelo geoelétrico (linha azul) de 58,2 $\mathrm{m}$ de profundidade, composto de cinco camadas inseridas manualmente e um substrato. $\mathrm{O}$ ajuste possui um erro de $4,12 \%$ (Figura 6 ) e a tabela 2 mostra os parâmetros do modelo geoelétrico: resistividade $(\rho)$, espessura da camada $(h)$, profundidade $(d)$ e altimetria $(A / t)$.

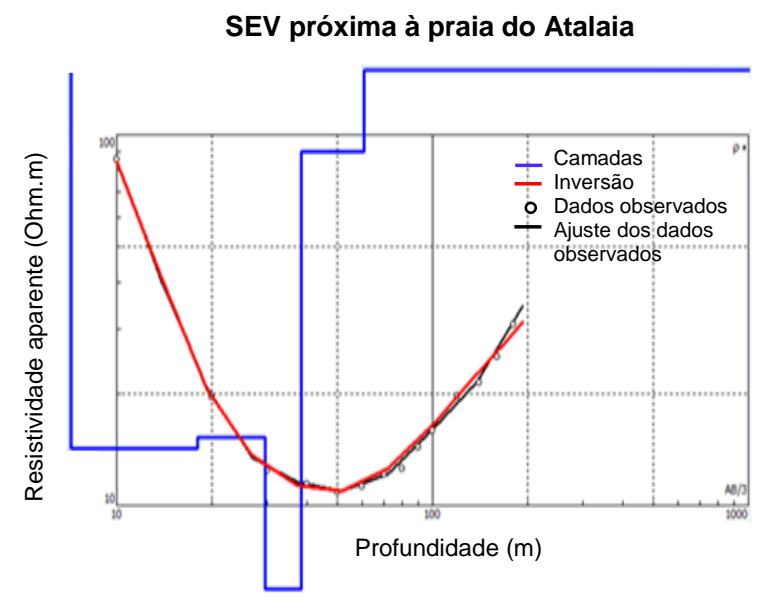

Figura 6 - Curva de resistividade próxima à praia do Atalaia.

Tabela 2- Resultado do modelo geoelétrico. $N$ : número de camadas, $\rho$ : resistividade, $h$ : espessura da camada, $d$ : profundidade e Alt: altimetria.

\begin{tabular}{l|c|c|c|c}
\hline 7 RMS $=4.12 \%$ & \multicolumn{1}{l}{} \\
\cline { 1 - 1 } $\mathbf{N}$ & $\mathbf{p}$ & $\mathbf{h}$ & $\mathbf{d}$ & Alt \\
\cline { 1 - 1 } $\mathbf{1}$ & $\mathbf{3 0 0}$ & $\mathbf{5}$ & $\mathbf{5}$ & $\mathbf{- 5}$ \\
\cline { 1 - 1 } $\mathbf{1}$ & $\mathbf{1 4 . 2}$ & $\mathbf{1 3}$ & $\mathbf{1 8}$ & $\mathbf{- 1 7 . 9 8}$ \\
\cline { 1 - 1 } $\mathbf{3}$ & $\mathbf{1 5 . 2}$ & $\mathbf{1 1 . 5}$ & $\mathbf{2 9 . 5}$ & $\mathbf{- 2 9 . 4 7}$ \\
\cline { 1 - 1 } $\mathbf{4}$ & $\mathbf{1 . 4}$ & $\mathbf{9}$ & $\mathbf{3 8 . 5}$ & $\mathbf{- 3 8 . 4 7}$ \\
\cline { 1 - 1 } $\mathbf{5}$ & $\mathbf{9 0}$ & $\mathbf{1 9 . 7}$ & $\mathbf{5 8 . 2}$ & $\mathbf{- 5 8 . 2 2}$ \\
\cline { 1 - 1 } $\mathbf{6}$ & $\mathbf{1 0 9 9}$ & & & \\
\hline
\end{tabular}

Com os resultados obtidos do modelo geoelétrico estabeleceu-se a correlação entre o perfil construtivo do poço próximo à praia do Atalaia e o modelo geoelétrico (Figura 7), para realizar a interpretação dos dados coletados.

O perfil do poço utilizado apresenta sete camadas, enquanto que o modelo geoelétrico cinco camadas e um substrato, isso se deve ao espaçamento inicial entre os eletrodos igual a $10 \mathrm{~m}$, impossibilitando delimitar camadas delgadas, como por exemplo, a primeira camada de $0,5 \mathrm{~m}$ de profundidade mostrada no perfil construtivo do poço.

O modelo geoelétrico apresenta uma camada rasa de 5 $\mathrm{m}$ de espessura e alta resistividade de 300 Ohm.m, correspondente a areia argilosa sem presença de fluidos da Formação Barreiras.

Da segunda camada à quarta camada corresponde a Formação Pirabas e o aquífero localiza-se de 5 a 57 m de profundidade, evidenciado devido aos baixos valores de resistividade elétrica. As camadas são constituídas por: areia calcífera (14,2 Ohm.m), calcário fraturado $(15,2$ Ohm.m) e areia calcífera (1,4 Ohm.m), respectivamente.

A quinta camada de 19,5 m de espessura, corresponde a rochas intemperizadas com resistividade de 90 Ohm.m, provenientes do embasamento cristalino e o substrato corresponde ao embasamento, com alta resistividade de 1099 Ohm.m, devido a sua constituição e ausência de fluidos.

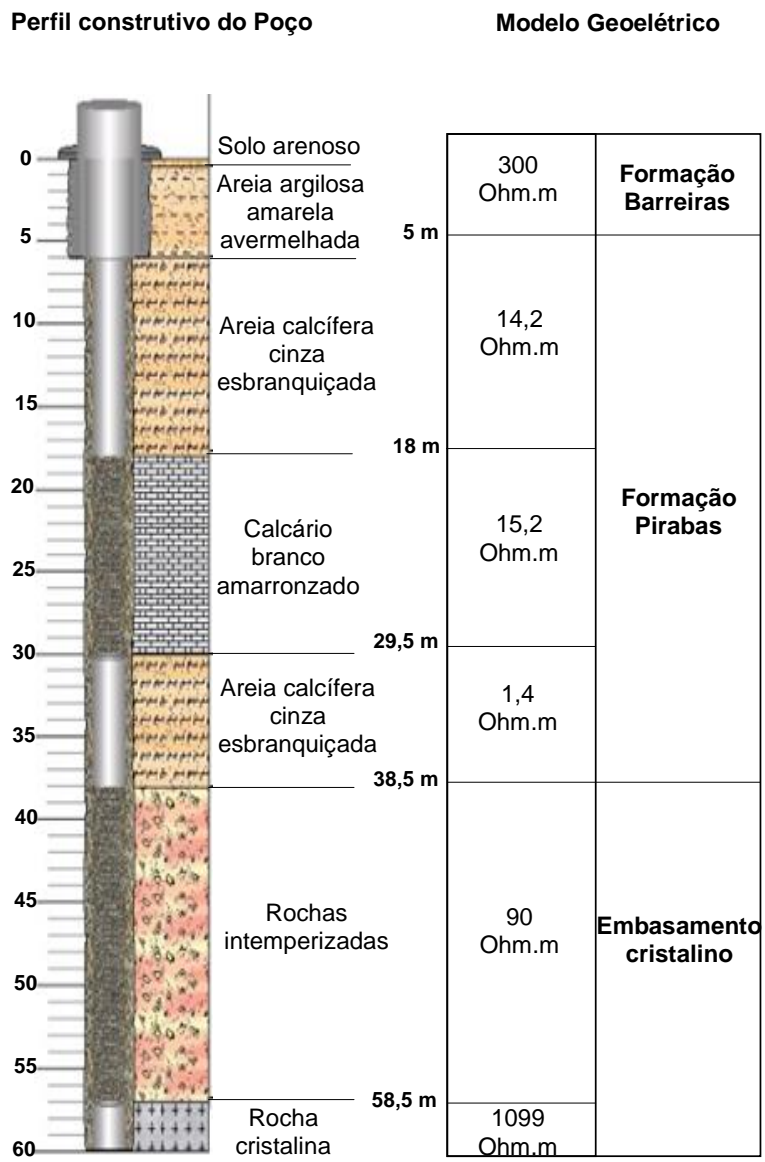

Figura 7 - Correlação entre o perfil construtivo do poço próximo à praia do Atalaia e o modelo geoelétrico.

\section{Conclusão}

A aplicação do método da eletrorresistividade no estudo de contatos geológicos costeiros em São João de Pirabas, na vila de Santa Luzia e em Salinópolis, próximo à praia do Atalaia, através de sondagens elétricas verticais (SEVs) mostrou-se satisfatório, rápido e econômico na ampliação de estudos geológicos e hidrogeológicos da região. Com o qual foi possível a partir de correlações entre dados geofísicos e de perfis de poços, identificar contatos entre as Formações e o embasamento cristalino, devido sua eficácia na caracterização em profundidade de contatos geológicos, 
desde que as litologias exibam forte contraste de resistividade elétrica.

Nos municípios de São João de Pirabas e Salinópolis os resultados estimaram que os contatos entre as Formações Barreiras e Pirabas localizam-se a aproximadamente $15 \mathrm{~m}$ de profundidade em São João de Pirabas e a $5 \mathrm{~m}$ de profundidade em Salinópolis, evidenciados devido aos valores dos contrastes de resistividade entre as unidades. Em Salinópolis, além de estimar o contato entre as Formações, foi possível identificar 0 topo do embasamento cristalino a aproximadamente $38 \mathrm{~m}$ de profundidade.

Os aquíferos subterrâneos identificados devido aos baixos valores de resistividade elétrica possuem $45 \mathrm{~m}$ de espessura em São João de Pirabas e 52 m de espessura em Salinópolis, localizados nas profundidades dos contatos entre as Formações Barreiras e Pirabas.

\section{Agradecimentos}

Ao Conselho Nacional de Desenvolvimento Científico e Tecnológico (CNPQ) pelo financiamento do projeto de estudo paleoambiental e paleogeográfico da Formação Pirabas (Paleógeno-Neógeno) com base em dados estratigráficos, paleontológicos e geofísicos, nordeste do estado do Pará, Brasil, que possibilitou a elaboração deste trabalho.

\section{Referências}

Costa, J. B. S., 1996. A neotectônica na Amazônia. In: Simpósio de geologia da Amazônia, vol.5, Boletim de resumos expandidos e guia de excursões. SBGeo-Núcleo Norte, Belém. p. 35-38.

Ferreira, C. S., 1966. Características lito-paleontológicas da Formação Pirabas, Estado do Pará. In: Conferência geológica das Guianas. p. 101-111.

Góes, A. M.; Rossetti, D. F.; Nogueira, A. C. R.; Toledo, P. M., 1990. Modelo deposicional preliminar da Formação Pirabas no nordeste do estado do Pará. Boletim do Museu Paraense Emilio Goeldi. Série Ciências da Terra, Belém, n.2, p. 3-15.

Loke, M. H., 1999. Electrical imaging surveys for environmental and engineering studies: a practical guide to. Penang, Malaysia: M. H. Loke. vol.2, p.57.

Petri, S., 1954. Foraminíferos fósseis da bacia de Marajó. Boletim da Faculdade de Filosofia, Ciências e Letras, Universidade de São Paulo (Geologia), vol.134, p. 1-172.

Petri, S., 1957. Foraminíferos Miocênicos da Formação Pirabas. Boletim da Faculdade de Filosofia, Ciências e Letras, Universidade de São Paulo (Geologia), vol. 216, p.1-79.

Rossetti, D. F., Truckenbrodt, W.; Góes, A. M., 1989. Estudo paleoambiental e estratigráfico dos sedimentos Barreiras e Pós-Barreiras na região bragantina, nordeste do Pará. Boletim do Museu Paraense Emílio Goeldi, Série Ciências da Terra, vol.1, p. 25-74.

Rossetti, D. F, 2000. Influence of low amplitude/high frequency relative sea-level changes in a wavedominated estuary (Miocene), São Luís Basin, northern Brazil. Sedimentary Geology, 133, n. 3, p. 295-324.

Rossetti, D. F., 2001. Late Cenozoic sedimentary evolution in Northeastern Pará, Brazil, within the context of sea level changes. Journal of South American Earth Sciences, vol.14, n.1, p. 77-89.

Rossetti, D. F; Góes, A. M; Ana, M.; Souza, Lena, S. B., 2001. Estratigrafia da sucessão sedimentar Pós Barreiras (Zona Bragantina, Pará) com base em radar de penetração no solo. Brazilian Journal of Geophysics, vol. 19.

Rossetti, D. F.; Góes A. M., 2004. O Neógeno da Amazônia Oriental Belém. Belém: Museu Paraense Emílio Goeldi. p. 13- 52.

Rossetti D. F.; Santos Jr. A. E. A, 2004. Facies architecture in a tectonically influenced estuarine incised valley fill of Miocene age, Northern Brazil. Journal of South America Earth Sciences, v. 17, p. 267-284.

Rossetti, D. F., 2006. Evolução sedimentar Miocenica nos estados do Pará e Maranhão. Revista do Instituto de Geociências-USP, São Paulo, vol.6, n. 2, p. 7-18. 